Utah State University

DigitalCommons@USU

$1-1-2013$

\title{
Test Validated Alignment and Stability Performance of the JMAPS Program Focal Plane Array Assembly in a Cryogenic Vacuum Environment
}

\author{
Brian C. Thompson \\ Pedro Sevilla \\ Mike Watson \\ Trent Newswander \\ Duane Miles \\ James Peterson
}

Follow this and additional works at: https://digitalcommons.usu.edu/sdl_pubs

\section{Recommended Citation}

Thompson, Brian C.; Sevilla, Pedro; Watson, Mike; Newswander, Trent; Miles, Duane; and Peterson, James, "Test Validated Alignment and Stability Performance of the JMAPS Program Focal Plane Array Assembly in a Cryogenic Vacuum Environment" (2013). Space Dynamics Lab Publications. Paper 131.

https://digitalcommons.usu.edu/sdl_pubs/131

This Article is brought to you for free and open access by the Space Dynamics Lab at DigitalCommons@USU. It has been accepted for inclusion in Space Dynamics Lab Publications by an authorized administrator of DigitalCommons@USU. For more information, please contact digitalcommons@usu.edu.

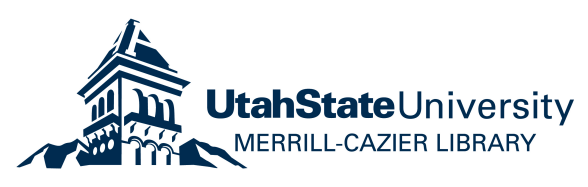




\title{
Test validated alignment and stability performance of the JMAPS program focal plane array assembly in a cryogenic vacuum environment
}

\author{
Brian C. Thompson, Pedro Sevilla, Mike Watson, Trent Newswander, Duane Miles, James Peterson \\ Space Dynamics Laboratory, 1695 North Research Park Way, North Logan, UT, USA 84335
}

\begin{abstract}
Focal Plane Arrays (FPA) consisting of multiple Sensor Chip Assemblies (SCA) in a precision aligned mosaic are being increasingly used in optical instruments requiring large format detectors. The Joint Milli-Arcsecond Pathfinder Survey Mission (JMAPS) requires very precise positional alignment and stability of its $2 \times 2$ SCA mosaic at operational temperatures to meet its precision sky mapping mission requirements. Key performance requirements include: detector active area co-planarity, in-plane alignment, and thermal stability. This paper presents an overview of the JMAPS Focal Plane Array Assembly, its alignment and thermal-mechanical stability requirements, and associated test-validated performance in a cryogenic vacuum environment.
\end{abstract}

Keywords: JMAPS, focal plane, sensor chip, mosaic, stability, thermal

\section{INTRODUCTION}

\subsection{JMAPS status and mission overview}

The JMAPS mission was a Department of Navy space-based, all-sky astrometric bright star survey, originally scheduled for launch in 2012. The primary goal of the JMAPS mission was to completely update the bright star astrometric, photometric, and spectroscopic catalogs covering a magnitude range of 1-12 with extended results through $15^{\text {th }}$ magnitude ${ }^{1}$. The planned orbit for the satellite was a $900 \mathrm{~km}$ sun-synchronous earth orbit.

The JMAPS program was re-baselined in October 2012, with development of major components of the JMAPS sensor moving forward under a technology demonstration effort. The Space Dynamics Laboratory (SDL) is currently integrating the JMAPS FPA assembly and optical telescope assembly. Electro-optical characterization testing of the integrated assembly is planned for completion during the summer of 2013.

\subsection{FPA assembly overview and driving requirements}

The FPA assembly (see Figure 1) consists of a 2 x 2 mosaic of Teledyne imaging sensor (TIS) H4RG-10 sensor chip assemblies mounted to a thermally and structurally stable mount ${ }^{2}$. The H4RG-10 is a 10 -micron pixel CMOS-Hybrid $\mathrm{SCA}^{2,3}$. The FPA assembly was designed, built, and tested by SDL.

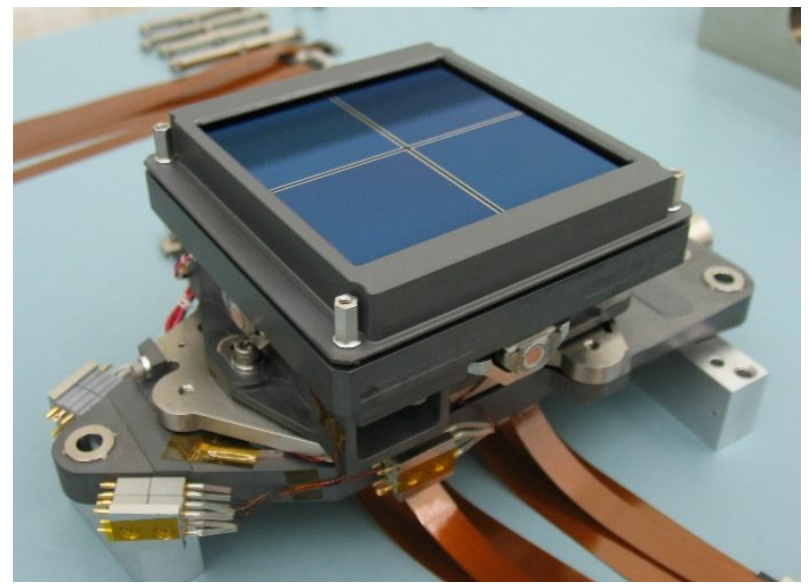

Figure 1. JMAPS FPA assembly.

Optomechanical Engineering 2013, edited by Alson E. Hatheway, Proc. of SPIE Vol. 8836, 88360 S · C 2013 SPIE · CCC code: $0277-786 X / 13 / \$ 18 \cdot$ doi: $10.1117 / 12.2024519$ 
Precise thermal control of the FPA assembly is required to meet mission accuracy requirements. The assembly is cooled to an operational temperature of $193 \mathrm{~K}$ via a thermal sink to a space radiator. Trim heating/cooling via thermo-electric coolers (TEC) provides thermal conditioning to a $0.010 \mathrm{~K}$ stability requirement over 45 minutes. The FPA assembly interfaces to three separate thermal zones which vary by up to $10 \mathrm{~K}$ over the stability time period.

Mission accuracy requirements also mandate precision alignment and mechanical stability of the SCA active areas at operational temperatures. The SCA active areas are aligned to: an out-of-plane co-planarity of $<10$ microns, in-plane adjacent SCA pixel spacing of $<2 \mathrm{~mm}$, differential SCA rotation of $+/-2$ milliradians, and opto-mechanical differential in-plane SCA closest edge approach stability of $<1.9 \mathrm{~nm}$.

The FPA assembly has a mass of $1.8 \mathrm{~kg}$ and it passed structural environmental tests which consisted of $45 \mathrm{~g}$ of quasistatic input and 9 grms of random-base input.

\section{TEST VALIDATED PERFORMANCE}

Performance of the FPA assembly driving requirements were validated via test in an operational environment.

\subsection{Co-planarity}

\subsubsection{Co-planarity test setup}

The co-planarity measurement test setup consists of a laser displacement meter mounted onto a three-axis stage. The test surfaces are scanned by moving the stages in plane and combining the displacement meter results into a 3D surface scan. Reference surface measurements are made continuously during a measurement and used to compensate for stage errors. The relatively long working distance of the laser displacement meter allows measurement of test surfaces through a vacuum window while at operational temperatures. Figure 2 shows a graphical depiction of the test setup.

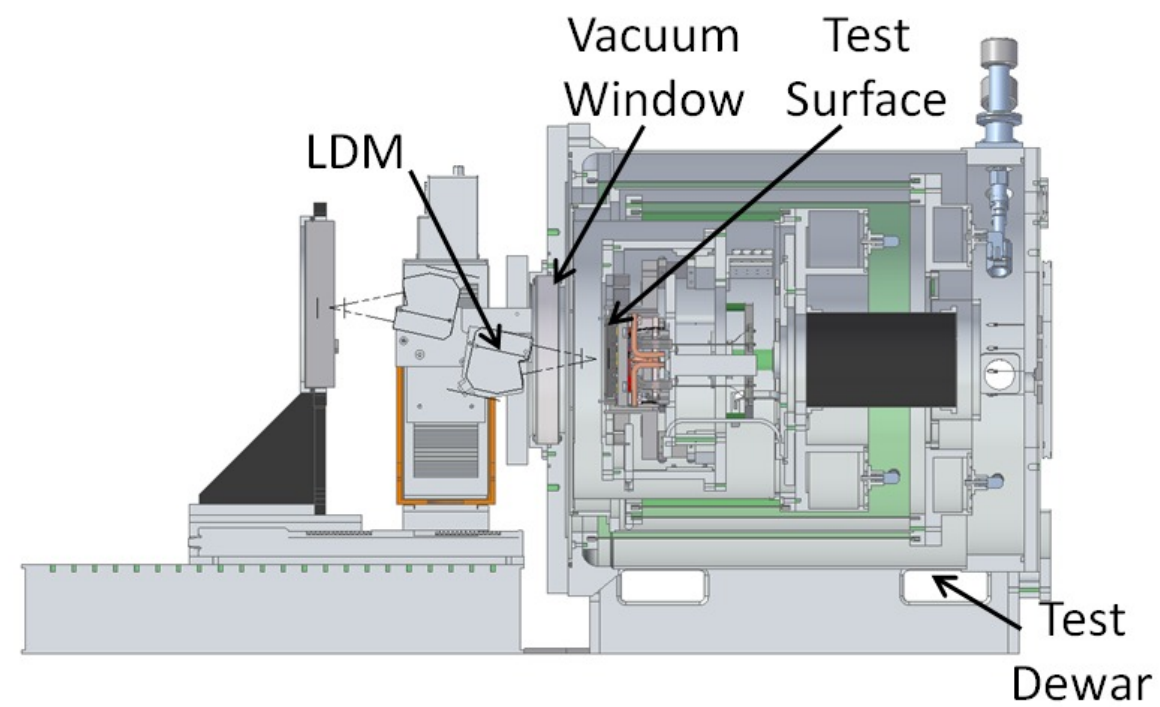

Figure 2. Co-planarity test setup.

\subsubsection{Co-planarity measurement uncertainty}

The co-planarity test setup measurement uncertainty was determined by scanning both flat and curved reference surfaces with known profiles at ambient and FPA assembly operational temperatures.

The team used optical flats with measured flatness much less than the co-planarity measurement resolution. These flats were scanned at both ambient and operational temperatures using the same test Dewar window used in the FPA assembly testing. No difference in flatness between ambient and operational temperature was observed. Typical measurement uncertainties of $+/-0.3$ to $+/-0.7$ microns were measured for flat profiles. 
The convex surface of a plano-convex lens with $5.002 \mathrm{~m}$ radius of curvature was also scanned at ambient temperatures. The convex surface provided a known surface measurement with departures from planarity at 340 microns compared to the 10 micron co-planarity requirement. The known surface profile was subtracted from the measured data. Figure 3 shows the resulting residual scatter plot with a measurement uncertainty of $+/-0.94$ microns. This magnitude is considered a worst-case bounding uncertainty for the co-planarity test setup.

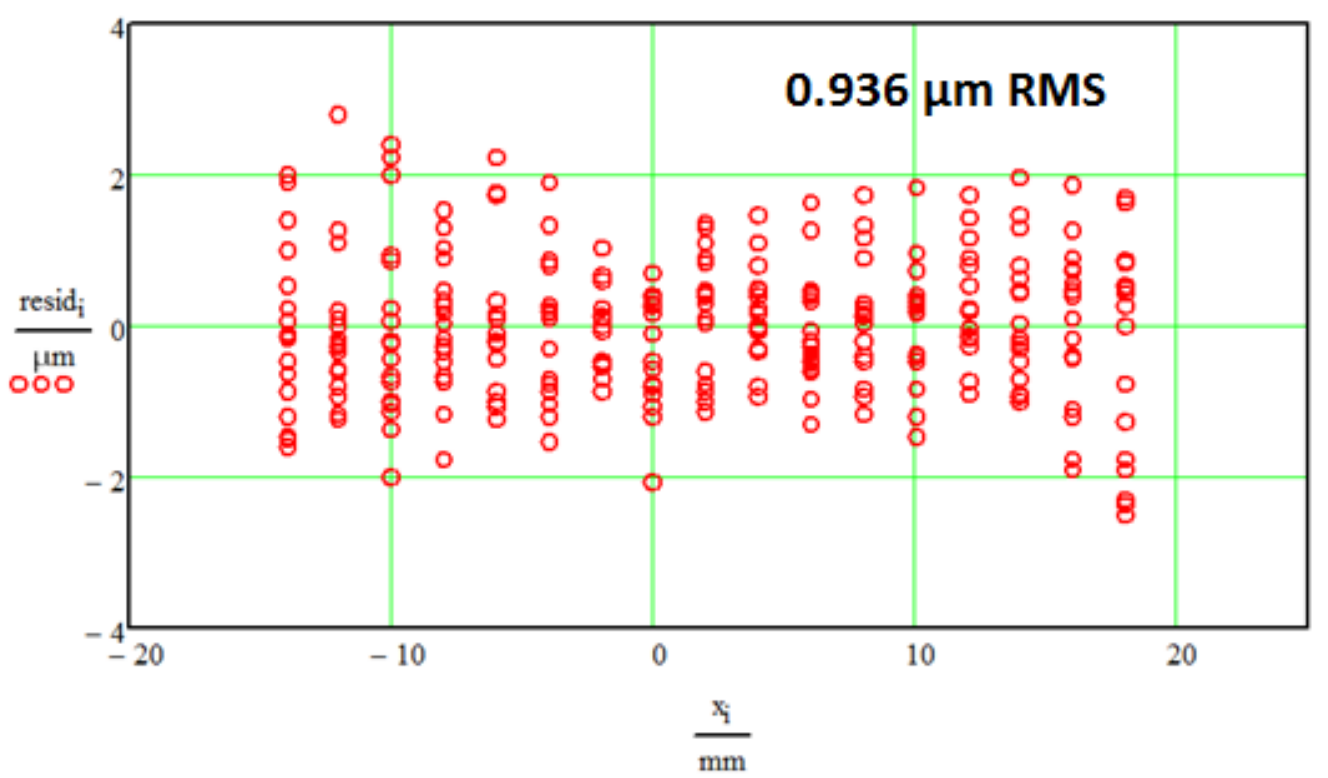

Figure 3. Worst-case co-planarity measurement uncertainty.

\subsubsection{Co-planarity results}

The FPA assembly showed excellent out-of-plane opto-mechanical stability throughout the test sequence. SCA coplanarity was measured following assembly and then at several test points throughout environmental testing (see Figures 4 through 6). Since the JMAPS FPA assembly co-planarity requirement applied only to the individual SCA active area best-fit planes, residuals (typically less than a few microns) from the best-fit planes were subtracted during postprocessing. Post-processed co-planarity measurements are reported as maximum minus minimum (peak-to-peak) profile values based on the individual SCA best fit planes. Figure 4 shows a 3D contour plot of the ambient temperature SCA co-planarity of 9.9 microns at the beginning of the test sequences.

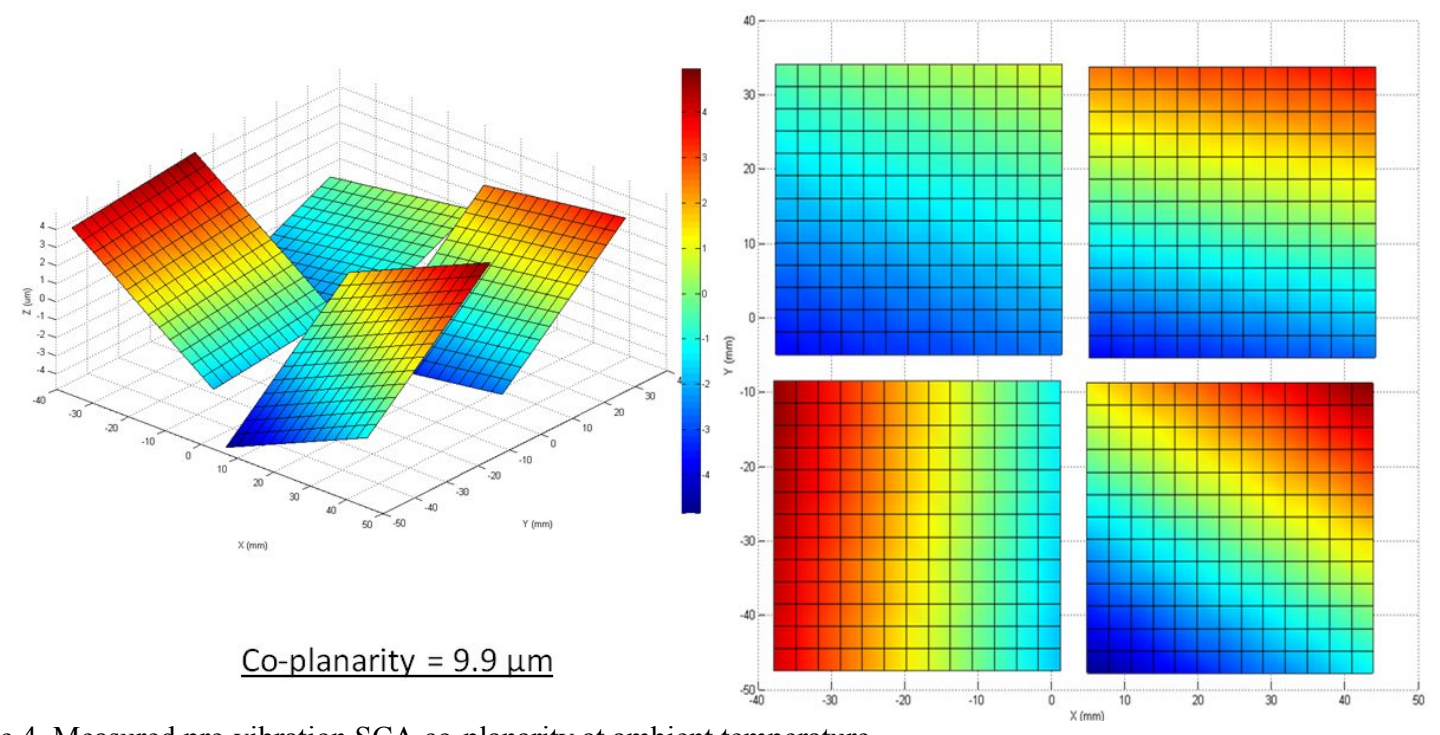

Figure 4. Measured pre-vibration SCA co-planarity at ambient temperature. 
After completion of vibration testing, the FPA assembly was cooled to operational temperatures resulting in a coplanarity of 9.8 microns (see Figure 5). At the conclusion of the testing, the co-planarity was measured at 10.3 microns (see Figure 6).
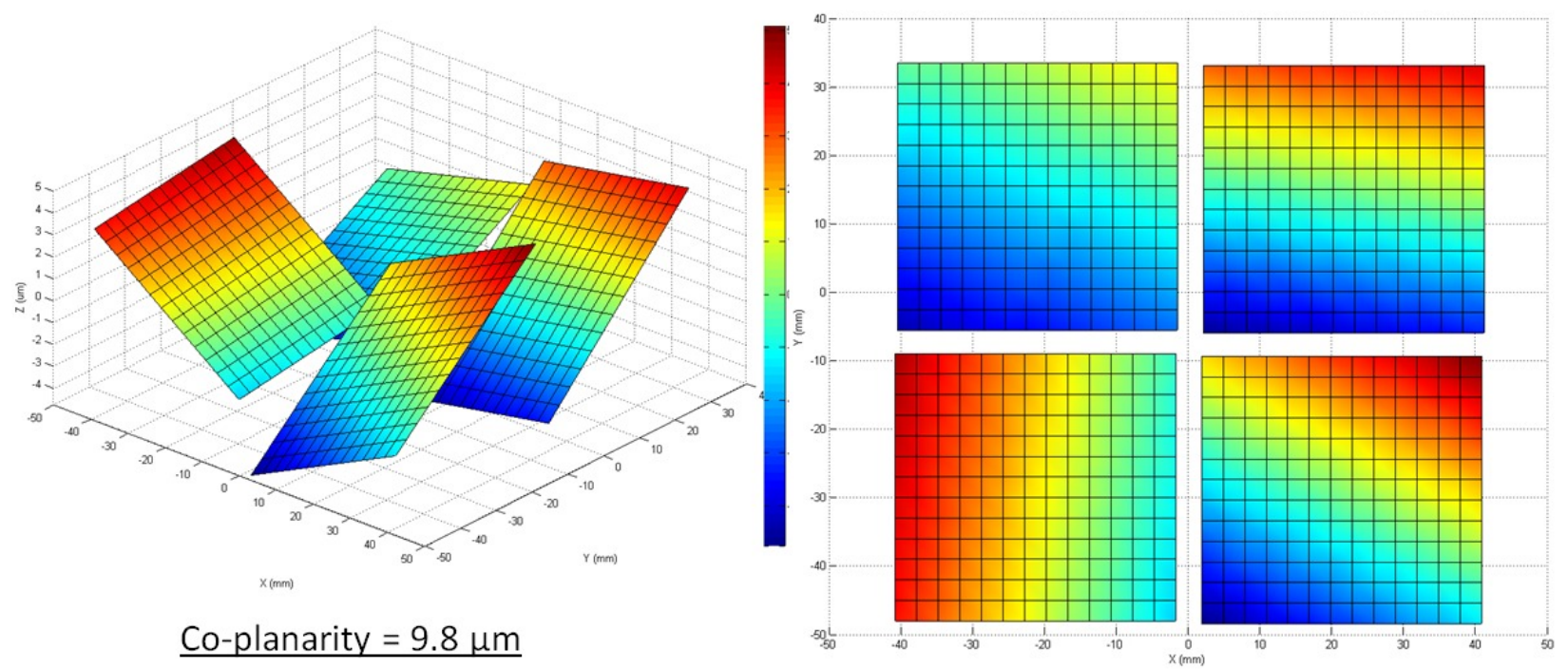

Figure 5. Measured post-vibration SCA co-planarity at $193 \mathrm{~K}$.

In addition to the results shown in this paper, other co-planarity measurements were performed during the testing and all results fell within the test setup measurement uncertainty of $+/-0.9$ microns.
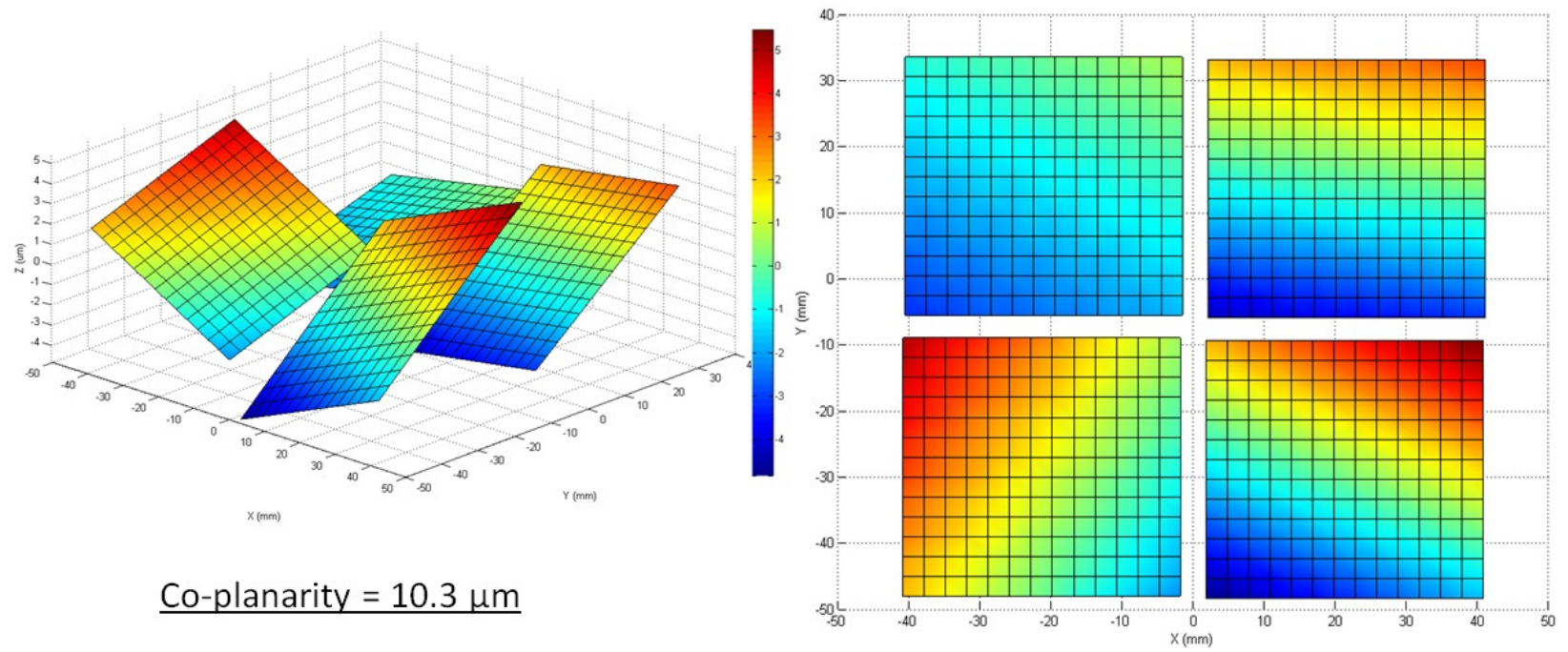

Figure 6. Measured post-vibration SCA co-planarity after returning to ambient temperature.

Due to funding limitations, schedule was not available to further improve the FPA assembly co-planarity performance. Additional iterations, using the SDL measurement and placement methods, could have produced co-planarity of less than 5 micrometers. However, since the FPA assembly met the co-planarity performance requirement, the use of additional funding and schedule resource to the incremental improvement was not justified.

\subsection{Thermal stability}

Thermal stability of the FPA assembly was test validated at operational conditions by simulating expected on-orbit transient thermal boundary conditions and measuring the controlled temperature stability of the test unit. The JMAPS mission thermal stability requirement was $<0.010 \mathrm{~K}$ over a 45 minute time frame. Worst-case transient fluctuations of 
the FPA assembly thermal interfaces were extracted from the system level thermal model. The following figures show the temporal variation in the FPA assembly radiator, telescope mount, and electronics thermal interfaces.

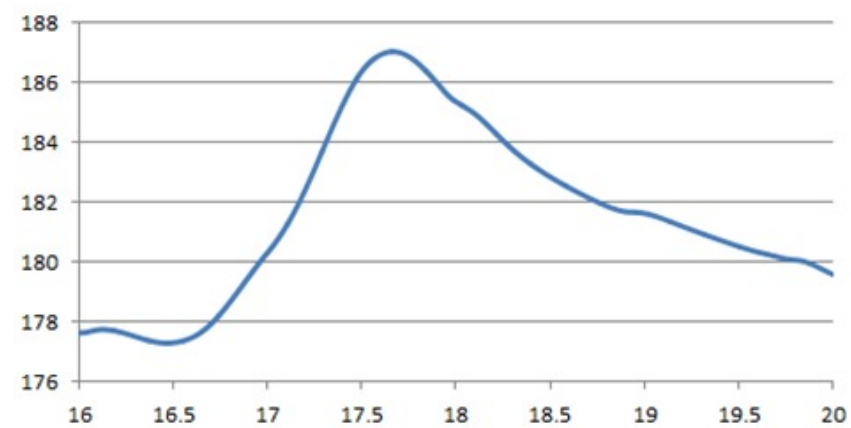

Figure 7. Radiator thermal interface temperature variation vs. time (K vs. hr.).

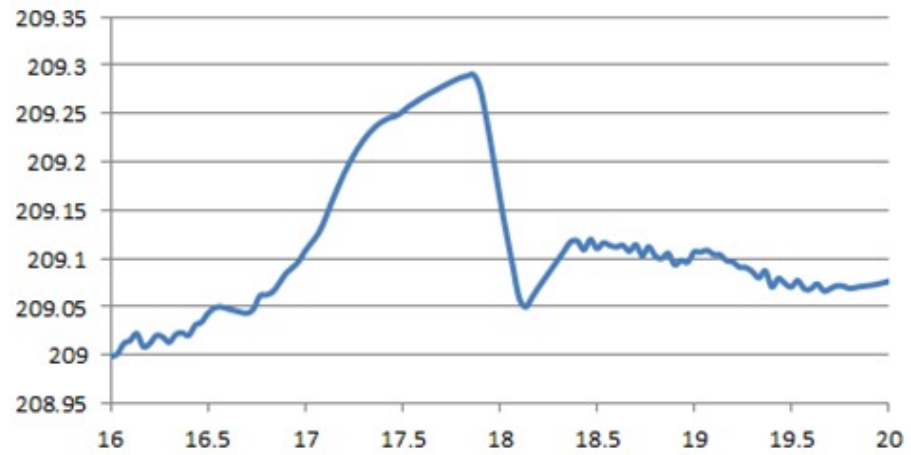

Figure 8 . Telescope mount thermal interface temperature variation vs. time (K vs. hr.).

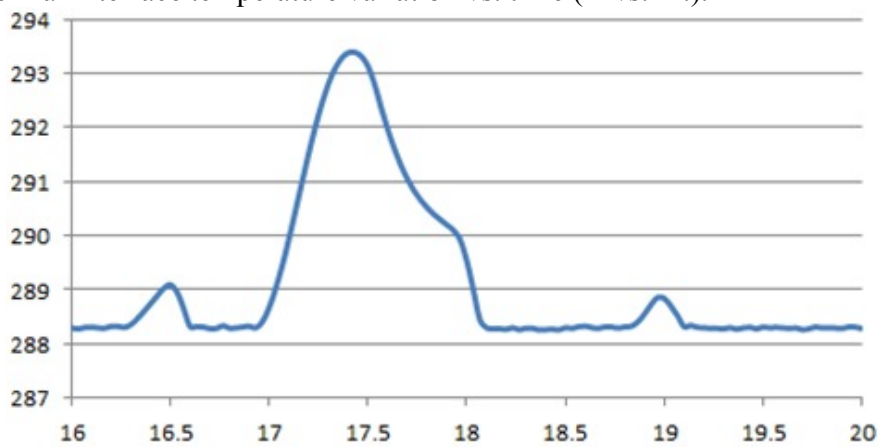

Figure 9. Electronics thermal interface temperature variation vs. time (K vs. hr.).

\subsubsection{Thermal stability test setup}

The thermal test setup included three independently controlled thermal zones to simulate the three thermal interfaces. The three zones were cold biased/cooled via flexible thermal links and heater controlled. Prior to conducting the tests, the controller for each zone was tuned to allow precise control of the thermal interface to the specified profile. The resulting thermal interface transients during test matched the specified profiles with no observable error.

\subsubsection{Thermal stability test results}

Figure 10 shows the test-validated thermal stability results for the FPA assembly. The four colored solid lines show the individual temperature responses of the four SCAs. The solid black is the average SCA temperature which served as the control input. Calculated thermal stability of the individual and average SCA responses over a 45 minute time frame are shown by the dashed lines. The temperature responses (solid lines) correspond to the left ordinate of the graph, and the stability results (dashed lines) correspond to the right ordinate. 


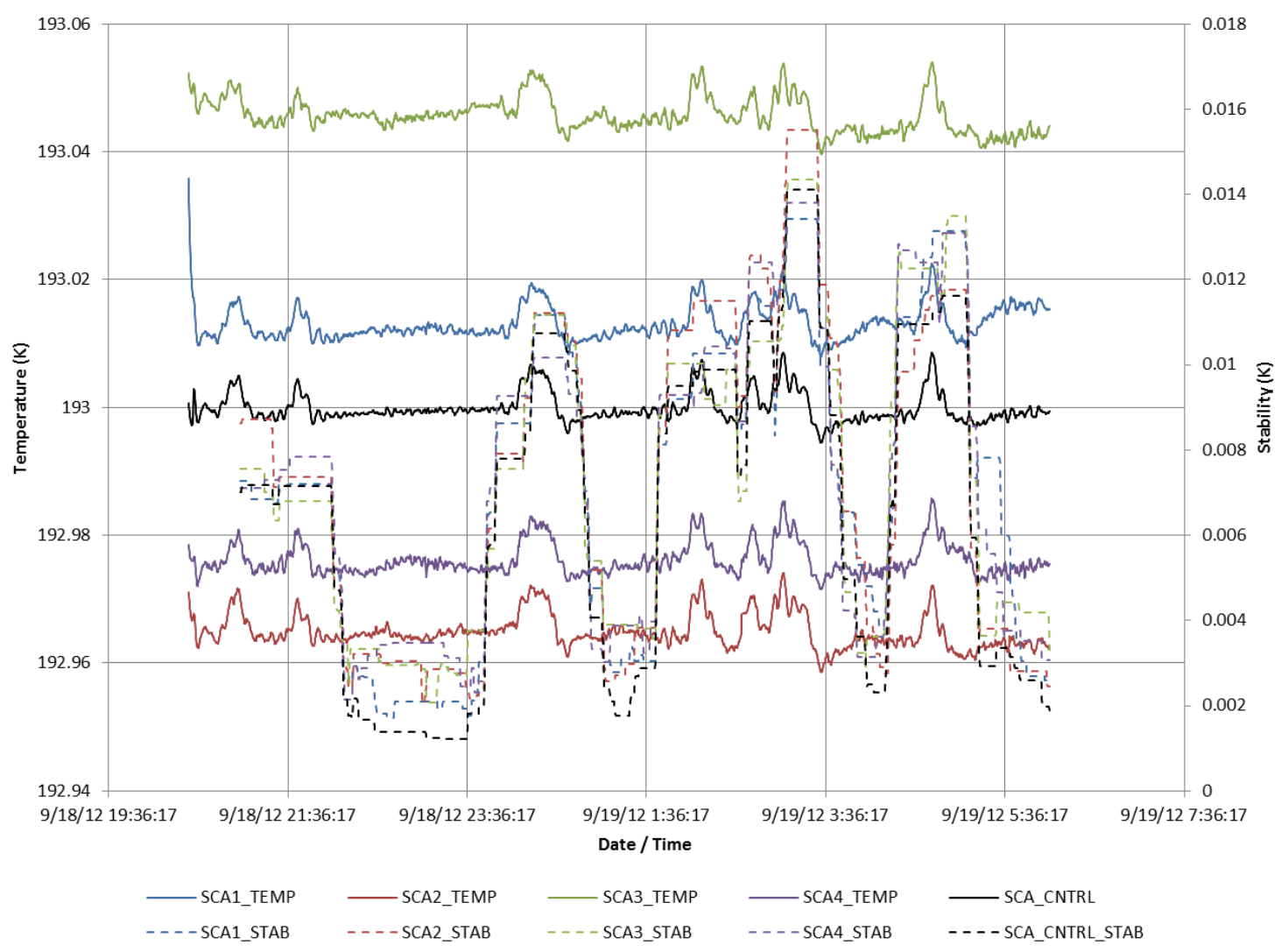

Figure 10. Thermal stability results (temperature and stability vs. time / date).

The FPA assembly showed excellent thermal stability results with worst-case performance of $<0.016 \mathrm{~K}$, which occurred only for a relatively short time during the eleven hour stability run. The majority of the time, the stability performance was $<0.010 \mathrm{~K}$.

A post-test assessment of the thermal stability results suggests that if a higher order controller was implemented, stability performance could be increased even further.

\subsection{In-plane alignment and stability}

The JMAPS mission requirements included many in-plane alignment and stability requirements which were all met by the FPA assembly. This publication focuses on three of the most challenging requirements: adjacent SCA pixel spacing of $<2 \mathrm{~mm}$, differential SCA rotation of $+/-2$ milliradians, and opto-mechanical differential in-plane SCA closest edge approach stability of $<1.9 \mathrm{~nm}$.

\subsubsection{Adjacent SCA pixel spacing and rotation}

To support a $<2 \mathrm{~mm}$ active area pixel spacing, the SCA packages were required to be placed within $0.1 \mathrm{~mm}$ of each other. After locating the SCAs, the resulting positions of the SCA pixels were measured at ambient and operational temperatures. The resulting ambient temperature adjacent SCA pixel spacing and rotation results are shown in Figure 11. 


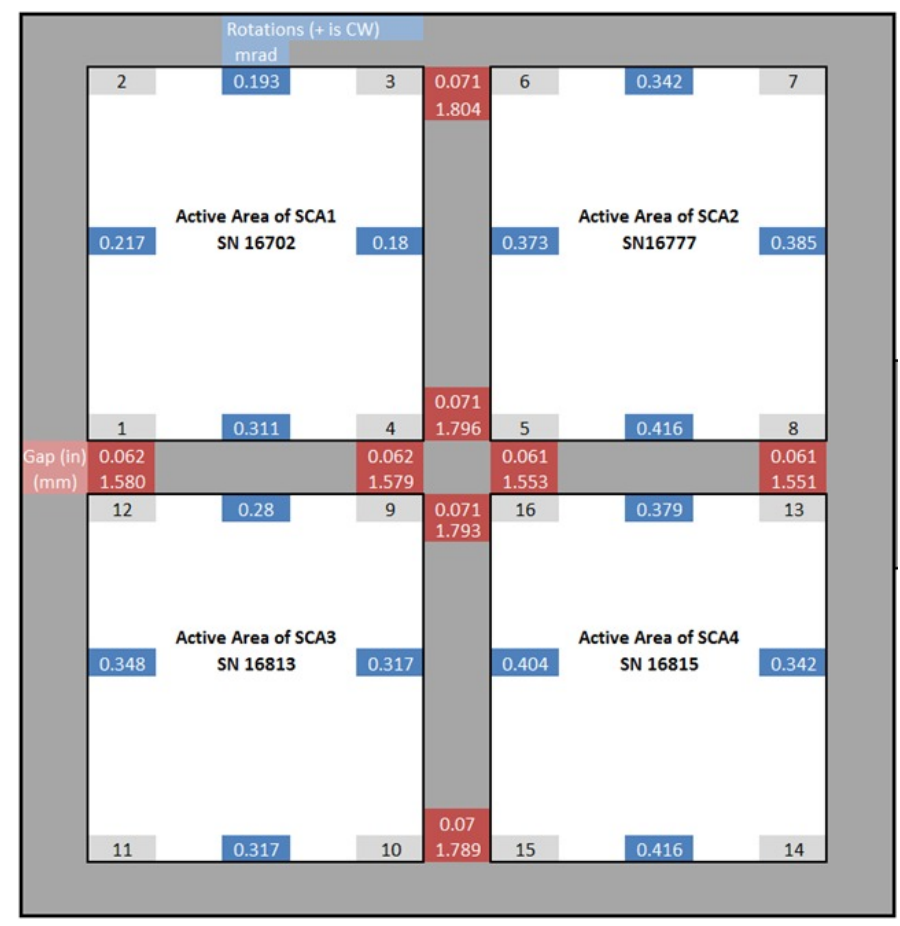

Figure 11. Ambient temperature measured adjacent SCA pixel space and rotations (not to scale).

Calculated adjacent SCA active area spacings are shown in red in both inches and millimeters with the maximum spacing at $1.8 \mathrm{~mm}$ versus the $2 \mathrm{~mm}$ requirement. Calculated relative SCA rotations are shown in blue with worst-case results of 0.4 milliradians versus the 2 milliradian requirement.

Ambient temperature spacing and rotation measurements were taken prior to and after completion of the test program (i.e. before and after vibration and thermal vacuum testing). The pre- and post-test SCA pixel spacings were with the measurement uncertainty of $0.005 \mathrm{~mm}$ indicating that the in-plane alignment did not change over the course of the testing.

SCA pixel spacing was also measured at operational temperatures by illuminating the focal planes with point sources of known spacing and back calculating the adjacent SCA pixel spacings and rotations. The results matched the ambient temperature results.

\subsubsection{Differential SCA closest edge approach stability}

The closest edge approach stability requirements specifies that the edges of the SCA packages cannot translate in-plane more than 1.9 nanometers over a 45 minute time frame. While directly measuring these small movements was not attempted, this requirement was verified via analysis and substantiated via the co-planarity measurements performed under operational conditions.

Verification by analysis was accomplished by performing a detailed structural optical thermal (STOP) analysis. A detailed thermal finite element model (FEM) of the FPA assembly was developed (see Figure 12). The same transient thermal inputs used for the thermal stability testing were applied to the thermal interface boundaries and the resulting transient gradients were calculated. 


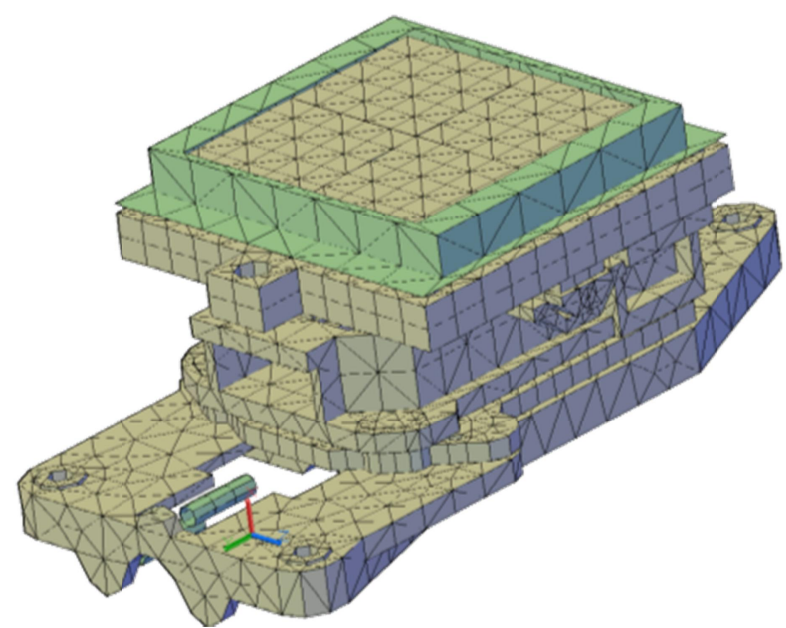

Figure 12. FPA assembly thermal finite element model (FEM).

Worst-case changes in thermal gradients over the 45 minute stability period were selected and transferred to the highly detailed opto-mechanical FEM and the resulting differential SCA edge distances were calculated. A snap shot of predicted SCA deformations at a selected time slice is shown in Figure 13. Note that the deformations are greatly exaggerated. For visual reference, the out-of-plane deformation of the four SCA active area surfaces is on the order of 2 microns.

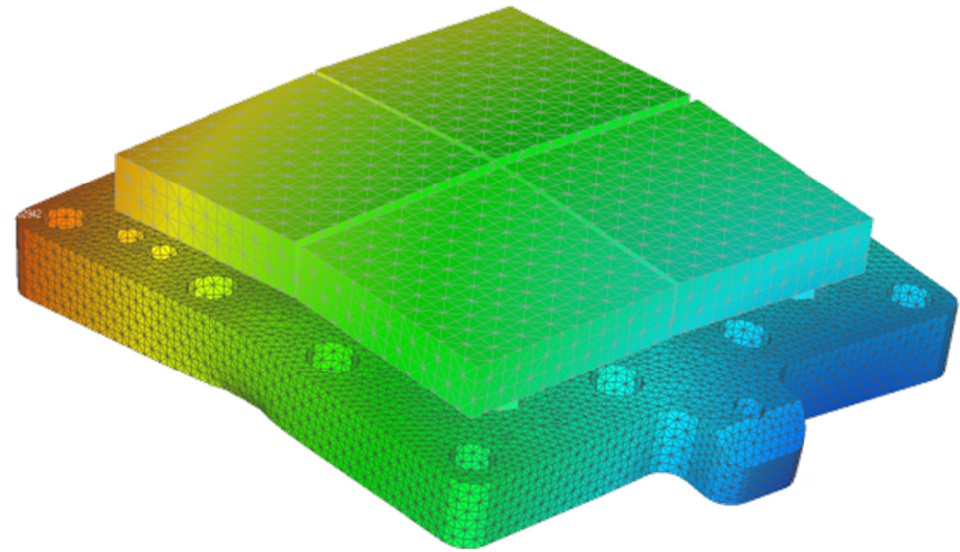

Figure 13. FPA assembly opto-mechanical FEM showing SCA edge deformations.

Changes in relative SCA edge separations were then calculated by subtracting the deformation over 45 minute time frames. The resulting worst change in differential in-plane SCA edge spacing over the 45 minutes was 1.5 nanometers compared to the 1.9 nanometer requirement.

In addition, the edge spacing stability requirement was corroborated by the co-planarity measurements at operational temperatures. The STOP analysis indicated that the majority of the change in SCA edge spacing was due to the SCA mount bending into a dome shape (see Figure 13) due to differential thermal expansion from ambient temperature to operational temperatures. The resulting magnitude of the "dome" was on the order of 2 micrometers, as previously stated. A comparison of the co-planarity results from ambient to operational temperatures show no measureable changes in co-planarity to an uncertainty of less than 1 micrometer. Since the doming shape was not observed during the coplanarity tests, it suggests that the STOP analysis results may be worst case.

\section{SUMMARY}

FPA assembly showed excellent test validated opto-mechanical and thermal alignment and stability performance. The co-planarity at the $193 \mathrm{~K}$ operational temperature was measured at 10 micrometers with potential for 5 micrometer 
performance for similar applications. Worst-case co-planarity measurement uncertainty was determined to be 0.94 micrometers with typical uncertainty estimated at 0.5 micrometers.

The FPA assembly thermal stability performance of $0.016 \mathrm{~K}$ over a 45 minute period applying transient thermal boundary inputs on the order of $10 \mathrm{~K}$ can readily be improved to $<0.010 \mathrm{~K}$ stability with potentially enhanced performance down to $0.005 \mathrm{~K}$ by applying a higher order controller.

In-plane alignment and stability also showed excellent performance. No changes in SCA locations to a 0.005 millimeter measurement uncertainty were observed from pre to post environmental testing. Differential SCA edge stability performance of 1.5 nanometers was verified by a high fidelity STOP analysis and correlated via the ambient and operational co-planarity measurements.

\section{ACKNOWLEDGMENTS}

The SDL JMAPS team would like to thank Bryan Dorland of the U.S. Naval Observatory and James Waterman of the Naval Research Laboratory for their support and the opportunity to work on this challenging mission. We would also like to thank Rachel Dudik of the U.S. Naval Observatory and Augustyn Waczynski of the Goddard Space Flight Center for their support and assistance with SCA testing. Also, many thanks to the entire SDL JMAPS team and support staff for their dedication, long days and nights, and hard work.

\section{REFERENCES}

[1] Dorland, B. N., Dudik, R. P., Dugan, Z. and Hennessy, G. S., "The Joint Milli-arcseond Pathfinder Survey (JMAPS) Mission Overview and Attitude Sensing Applications," AAS 09-181 (2009).

[2] Dorland, B. and Dudik, R, "Joint-Milli-Arcsecond Pathfinder Survey (JMAPS): Overview and Application to NOW Mission," 11 March 2009, <http://newworlds.colorado.edu/documents/ASMCS_Report/nwo_H_jmaps.pdf $>$ (22 June 2013).

[3] Teledyne Imaging Sensors H4RG Visible \& Infrared Focal Plane Array, April 2012, < http://www.teledynesi.com/imaging/H4RG\%20Brochure\%20-\%20rev3\%20v2-2\%20-\%20OSR.pdf $>$ (22 June 2013). 